\title{
The TANGO Project: Thorough ANalysis of radio-Galaxies Observations
}

\author{
Breezy Ocaña Flaquer ${ }^{1}$, Stephane Leon Tanne ${ }^{2}$, \\ Francoise Combes ${ }^{3}$, and Jeremy Lim $^{4}$ \\ ${ }^{1}$ Instituto de Radio Astronomía Milimétrica, Av. Divina Pastora 7, bloque 20, Granada 18012, \\ Spain \\ Email: ocana@iram.es \\ ${ }^{2}$ Instituto Astrofísico de Andalucía, Camino Bajo de Hutor 50, Granada E-18008, Spain \\ Email: leon@iram.es \\ ${ }^{3}$ Observatoire de Paris, LERMA, 61 Av. de l'Observatoire, F-75014 Paris, France \\ Email: francoise.combes@obspm.fr \\ ${ }^{4}$ Department of Physics, The University of Hong Kong, Pokfulam Road, Hong Kong \\ Email: jjlim@hku.hk
}

\begin{abstract}
We present a sample of radio galaxies selected only on the basis of radio continuum emission and we confirm that these galaxies have lower molecular gas mass than other elliptical galaxies with different selection criteria.
\end{abstract}

Keywords. galaxies: ISM, radio continuum: galaxies

The TANGO project studies the properties of radio galaxies selected only on the basis of their radio continuum emission (see Ocana Flaquer et al. 2009, for a detailed analysis). The sample is divided in two groups, 52 galaxies at low redshift ( $\mathrm{LzS}, 0<z<0.1$ ) and 49 galaxies at medium redshift $(\mathrm{MzS}, 0.3<z<1.0)$. We have worked on the LzS so far, observing $\mathrm{CO}(1-0)$ and $\mathrm{CO}(2-1)$ with the IRAM 30-m telescope and conclude that these galaxies have a lower molecular gas mass $\left(\sim 10^{8} M_{\odot}\right)$ than other samples $\left(>10^{9} M_{\odot}\right)$ with different selection criteria, such as an FIR-selected sample (e.g., Evans et al. 2005). Even if our sample has a low molecular gas content, it is able to host an AGN down to a mass of about $10^{6} M_{\odot}$.

Galaxies in TANGO follow the radio galaxy type classification (FR I and FR II) proposed by Fanaroff \& Riley (1974). The LzS shows that the galaxies tend to be FR I and the galaxies further away tend to be FR II, but in general FR II galaxies have a higher molecular gas mass $\left(5 \times 10^{8} M_{\odot}\right)$ than FR I galaxies $\left(2 \times 10^{8} M_{\odot}\right)$.

We find that $L_{\mathrm{CO}}$ vs. $L_{\mathrm{FIR}}$ shows a linear relation typically interpreted in spiral galaxies as indicating star formation. If we compare the FIR flux ratio $f_{100} / f_{60}$, which according to Bell (2003) suggests where is the IR coming from, to those of other samples, the $\mathrm{LzS}$ has a ratio of 1.9 suggesting that the galaxies have a high temperature heated up by young stars in $\mathrm{H}$ II regions. Nevertheless we cannot exclude an AGN contribution to heating of the dust.

\section{References}

Bell, E. F. 2003, ApJ, 586, 794

Evans, A. S., et al. 2005, ApJS, 159, 197

Fanaroff, B. L. \& Riley, J. M. 1974, MNRAS, 167, 31P

Ocana Flaquer, B., Leon, S., Combes, F., \& Lim, J. 2009, $A \& A$, in press 